\title{
ADHERENCIA A LA QUIMIOTERAPIA Y RADIOTERAPIA EN PACIENTES ONCOLÓGICOS: UNA REVISIÓN DE LA LITERATURA
}

\author{
ADHERENCE TO CHEMOTHERAPY AND RADIOTHERAPY IN PATIENTS WITH \\ CANCER: A REVIEW OF THE LITERATURE
}

Ximena Palacios-Espinosa, Laura P. Vargas-Sterling

Universidad del Rosario, Bogotá, Colombia

Resumen

Objetivo: Realizar una revisión de la literatura sobre la adherencia a la quimioterapia y a la radioterapia para el cáncer, por los altos costos biopsicosociales y culturales que acarrean y que están relacionados con bajas tasas de adherencia. Método: se realizó una búsqueda de la literatura en bases de datos especializadas utilizando las palabras clave. Resultados: Debido a que existe un porcentaje significativo de pacientes que no se adhieren a los tratamientos oncológicos y a que las consecuencias de no hacerlo pueden desencadenar un mayor riesgo de muerte o progresión de la enfermedad, es fundamental abordar este tema y para esto tener en cuenta las diferentes variables de tipo biológico, psicológico y social que influyen en la conducta de adherencia o no adherencia a los tratamientos, entre ellas la experimentación de efectos secundarios, la motivación, la capacidad de toma de decisiones, el estado emocional, la autonomía y la filosofía de vida de cada paciente. Esta investigación contribuye a que el personal de salud tenga más elementos para abordar esta problemática y así sus intervenciones sean más efectivas pudiendo incrementar la adherencia en los pacientes. Conclusiones: La adherencia es un constructo multifactorial y multideterminado, ampliamente estudiado en tratamientos no oncológicos (p.e. SIDA) y hay poca investigación que avale la eficacia de las intervenciones psicológicas para abordar la no
Abstract

Objective: To review in databases the relevant literature of adherence to chemotherapy and radiotherapy for cancer; which bring high biological, psychological, social and cultural costs and have been related to lower adherence rates. Method: A search in different databases was performed to identify relevant articles related to the key words. Results: A significant percentage of patients do not adhere to cancer treatments and the consequences of not doing it can unleash in a major risk of death or disease progression, then it is essential to approach this topic and take into account different variables of biological, psychological and social impact on the behavior of adherence or not adherence to the treatments; including the side effects, motivation, decision-making ability, emotional condition, autonomy and life philosophy of each patient. This research contributes to the health staff have more elements to approach this problem and so his interventions are more effective being able to increase the patient's adherence. Conclusions: Adherence is a multifactorial and multi-determined construct, which has been extensively studied in non cancer treatments (e.g. AIDS) and there is few investigation that supports the efficacy of the psychological interventions to approach not adherence and to promote the adherence to cancer treatments.

\section{Correspondencia}

Ximena Palacios-Espinosa

Dirección de contacto: Carrera 24 \# 63C - 69,

Bogotá, D. C., Colombia

E-mail: ximena.palacios@urosario.edu.co; lauravargass@hotmail.com 
adherencia y promover la adherencia a tratamientos oncológicos.

Palabras clave: Adherencia, quimioterapia, radioterapia, cáncer, evaluación, tratamiento, factores de riesgo.
Keywords: Adherence, chemotherapy, radiotherapy, neoplasms, evaluation, treatment, risk factors.

\section{PLANTEAMIENTO DEL PROBLEMA}

Tres son los tratamientos que tradicionalmente se han utilizado para el manejo del cáncer: (1) la cirugía, (2) la radioterapia y (3) la quimioterapia. Suelen administrarse de forma conjugada o seleccionarse uno u otro de ellos, de acuerdo con las características del caso clínico a $\operatorname{tratar}^{(1)}$.

Sin embargo, a pesar de los importantes avances científicos en la administración de cada uno de ellos, siguen siendo iatrogénicos e implican un alto costo psicológico y biológico para los pacientes ${ }^{(2,3)}$. Por ejemplo, aún si la tendencia es que la cirugía oncológica sea de carácter conservador, las mutilaciones visibles y no visibles continúan predominando; pese a la existencia de perfeccionadas y novedosas moléculas antieméticas, la radioterapia y la quimioterapia continúan provocando náuseas y vó$\operatorname{mitos}^{(4,62)}$ que interfieren en la cotidianidad y el bienestar subjetivo de los pacientes. Sin embargo, el propósito de todo tratamiento oncológico es reducir el volumen tumoral e idealmente erradicar la masa o lesiones metastásicas; la cirugía a través de la resección, la radioterapia a través de la radiación y la quimioterapia a través de la administración de agentes antineoplásicos; pero los deletéreos efectos colaterales que se pueden presentar como resultado de los tratamientos oncológicos mencionados, ponen en riesgo la probabilidad de adherencia del paciente a ellos. En este artículo, se abordará el problema de la adherencia a quimioterapia y radioterapia.

Mientras la radioterapia es localizada, la quimioterapia es sistémica ${ }^{(1)}$. Sin embar- go, ambas producen efectos colaterales ${ }^{(63-64)}$ (nauseas, vómito, diarrea, mucositis, inmunosupresión, alopecia, astenia, adinamia, etc.(1) cuya intensidad depende de la zona irradiada, de la dosis de radiación o de medicamento, del tipo de medicamento utilizado, de las diferencias individuales, de la competencia inmunológica, entre otros. Lo anterior explica que los tratamientos oncológicos hayan sido históricamente estigmatizados pues la experiencia de los pacientes que los reciben, ha trascendido a la población general resaltándose la desagradable y costosa experiencia con ellos. Así, los prejuicios y los imaginarios colectivos alrededor de dichos tratamientos pueden explicar las respuestas de evitación que emiten quienes deben decidir el inicio o mantenimiento del tratamiento oncológico. Pero si bien es cierto que los efectos secundarios se presentan con alta frecuencia, también lo es que los avances científicos han favorecido el desarrollo de diversidad de medicamentos y medidas terapéuticas paliativas para ellos, que pueden hacer soportable la convivencia con el tratamiento oncológico. Además, es de fundamental importancia considerar que las respuestas de evitación, el rechazo del tratamiento o la baja adherencia al mismo, pueden desencadenar en un mayor riesgo de muerte o de progresión de la enfermedad.

Todo lo anterior pone en evidencia la relevancia de conocer cuáles son los aspectos que están relacionados con la adherencia al tratamiento oncológico. En consecuencia, es conveniente realizar esta investigación teórica pues existe un porcentaje significativo de pacientes que no 
se adhieren al tratamiento oncológico y por lo tanto es importante conocer cuáles son las posibles razones para ello. Esto puede contribuir a que el personal de salud tenga más elementos para abordar esta problemática y por tanto, mejorar algunos aspectos que disminuyen la probabilidad de adherirse al tratamiento. Se podrá dar aviso al personal de salud sobre aquellos factores que impiden o que favorecen que un paciente se adhiera al tratamiento de tal manera que se puedan desarrollar futuras intervenciones más efectivas para incrementar la adherencia. Además, con esta investigación teórica se puede resaltar la importancia de realizar más investigaciones relacionadas con la adherencia al tratamiento específicamente a la radioterapia y a la quimioterapia debido a que no se han realizado muchas en relación con pacientes oncológicos pero sí con otro tipo de enfermedades ${ }^{(5)}$.

Esta revisión se enmarca teóricamente en el modelo biopsicosocial y la Psicología de la Salud, y se respalda en una perspectiva cognoscitivo comportamental. El primero, permite resaltar la importancia de factores psicológicos, biológicos y sociales y su relación con la adherencia al tratamiento de los pacientes con cáncer que reciben quimioterapia y radioterapia específicamente. Por su parte, la perspectiva cognoscitivo comportamental es sólo un camino para conceptualizar la problemática de estudio y está fundamentada en suficiente literatura que demuestra que factores conductuales, cognoscitivos y afectivos contribuyen en el comportamiento humano ${ }^{(6)}$ (p. 574). De hecho esta perspectiva plantea que: (1) las personas somos procesadores activos de la información, más que reactores pasivos a las contingencias ambientales; (2) los pensamientos (evaluaciones, atribuciones, expectativas) pueden elicitar o modular el afecto y la activación fisiológica porque ambos son motivadores de la conducta; (3) la con- ducta está recíprocamente determinada por el ambiente y por el individuo; (4) si las personas han aprendido pensamientos, conductas y sentimientos maladaptativos, las intervenciones deberán dirigirse a ellos en conjunto; (5) ya que las personas son instrumentos en el desarrollo y mantenimiento de pensamientos, sentimientos y conductas maladaptativos, pueden ser, son y serán agentes activos de cambio de su conducta maladaptativa $^{(6)}$ (p. 577).

\section{OBJETIVOS}

El objetivo de este artículo fue realizar una revisión de la literatura que permitiera conocer el estado actual del conocimiento sobre la adherencia a la quimioterapia y a la radioterapia para el cáncer. Para ello, se tuvieron en cuenta los siguientes objetivos específicos: (1) definir conceptualmente adherencia, (2) identificar las tasas de deserción del tratamiento oncológico, (3) los factores biológicos, psicológicos, sociales y culturales que pueden aumentar la probabilidad de adherencia al tratamiento oncológico, (4) los factores biológicos, psicológicos, sociales y culturales que pueden disminuir la probabilidad de adherencia al tratamiento oncológico y (5) los métodos y técnicas de evaluación e intervención para los problemas de adherencia al tratamiento oncológico.

\section{DEFINICIÓN DEL CONCEPTO “ADHE- RENCIA"}

La adherencia al tratamiento se define como "el grado en que la conducta de un paciente, en relación con la toma de medicación, el seguimiento de una dieta o la modificación de hábitos de vida, se corresponde con las recomendaciones acordadas con el profesional de la salud" (7).

Se considera que hay adherencia al tratamiento cuando no hay retraso de éste ${ }^{(8)} \mathrm{O}$ no hay interrupción temprana del mismo(9). 
The International Society for Pharmacoeconomics and Outcome Research (ISPOR) definió recientemente adherencia como un sinónimo de cumplimiento, es decir "el grado de conformidad con las recomendaciones sobre el tratamiento del día a día por el proveedor con respecto a la fecha, la dosis, y la frecuencia ${ }^{(10)}$.

Según Alfonso(11), los conceptos alianza, cooperación y adherencia parecen permitir una mayor participación del paciente en la toma de decisiones que afectan a su propia salud. Se supone que el paciente se adhiere a un plan con el que está de acuerdo y en cuya elaboración ha podido contribuir o al menos ha aceptado la importancia de realizar acciones concretas que se incluyen en el programa a poner en práctica, de ese modo involucraría una consideración activa de la persona.

Tradicionalmente, el personal médico ha utilizado el término cumplimiento para referirse a los diferentes comportamientos adoptados por los pacientes al momento de recibir las órdenes impartidas por su médico tratante. Sin embargo debido a que este término connota una obediencia renuente, muchos psicólogos de la salud y algunos médicos prefieren utilizar otros términos como adherencia, obediencia, colaboración, cooperación. Se plantea que este último es el ideal, ya que tanto el profesional de la salud como el paciente se preocupan por la recuperación y el mantenimiento de la salud del paciente. Sin embargo, debido a que ésta no es una práctica común, se ha llegado a la conclusión de que los términos cumplimiento y adherencia siguen siendo las palabras de mayor uso ${ }^{(12)}$. También se ha reconocido la persistencia como un término estrechamente asociado con la adherencia en la medida en que se refiere al tiempo que dura la terapia desde que inicia hasta que finaliza(10).

En consecuencia con lo anterior, se puede afirmar que no hay adherencia al tratamiento cuando los pacientes no se toman sus medicamentos correctamente, olvidan o se niegan a seguir una dieta, no realizan los ejercicios prescritos, cancelan o no acuden a las citas y persisten en estilos de vida que ponen en peligro su salud $^{(13)}$. La no adherencia significa, evitar, olvidar o dejar de tomar los medicamentos o la tendencia de alterar o ajustar las dosis recomendadas por el médico ${ }^{(14)}$.

Específicamente en el tratamiento con quimioterapia se define la no adherencia cuando hay reducción de la dosis de infusión ${ }^{(8)}$. Por ejemplo, en un estudio que se realizó con pacientes con cáncer de seno que recibían quimioterapia adyuvante, se consideró que las personas no eran adherentes si: (a) cumplían con menos de 6 ciclos de quimioterapia, teniendo en cuenta que el objetivo eran 8 ciclos; (b) si se reducía la dosis en más del 25\% de los ciclos y (c) si se interrumpía el tratamiento debido a la negativa del paciente o a la toxicidad de la quimioterapia ${ }^{(15)}$. Otro ejemplo concreto de no adherencia a la quimioterapia lo presentan ${ }^{(16)}$ en un estudio realizado con niños con cáncer. En este se consideró que no hubo adherencia al tratamiento cuando los pacientes no recibían sus medicamentos a tiempo o en las dosis apropiadas y cuando se perdía más del $10 \%$ de las citas médicas programadas.

De acuerdo con lo anterior, se puede afirmar que no adherencia es cuando el incumplimiento es suficiente para interferir considerablemente con la consecución del objetivo terapéutico ${ }^{(17)}$.

En conclusión, el concepto de adherencia tiene múltiples significados. Diferentes autores proponen sinónimos que hacen referencia al mismo concepto, pero vale la pena resaltar que estos conceptos se relacionan unos con otros y a la vez se complementan, con el objetivo de dar una definición más completa. No obstante, en el presente artículo se conservará el término adherencia. 


\section{TASAS DE DESERCIÓN Y DE ADHERENCIA AL TRATAMIENTO ONCOLÓGICO}

Cerca de la mitad de los pacientes en los Estados Unidos no se adhieren a las recomendaciones de sus médicos para la prevención o tratamiento de las enfermedades crónicas o agudas ${ }^{(13)}$. La literatura sugiere que entre el $25 \%$ y el $50 \%$ de los pacientes no cumplen con las recomendaciones médicas, incluyendo tomar los medicamentos prescritos, asistir a las citas y modificar hábitos personales ${ }^{(18)}$.

Las tasas de adherencia a las terapias con medicamentos suministrados por largo tiempo, como puede ser el caso de la quimioterapia oral a menudo administrada para el tratamiento de algunos tipos de cáncer, son sorprendentemente bajas $(40 \%-50 \%)$ o variables $(20 \%-100 \%)^{(19-20)}$. Incluso se calcula que 1 de cada 10 pacientes abandonan la primera prescripción de la quimioterapia oral $\left.{ }^{(71}\right)$. Por ejemplo, las tasas de adherencia a las terapias por vía oral varían del $16 \%$ al $100 \%$ en pacientes con cáncer de mama ${ }^{(21)}$. Específicamente con respecto a la adherencia al Tamoxifeno o al inhibidor aromatasa, del $23 \%$ al $28 \%$ de los pacientes con cáncer de seno interrumpieron su terapia hormonal oral antes de lo recomendado ${ }^{(22)}$.

Según estudios publicados entre 1948 y 1998 , existe un porcentaje del $25 \%$ de no adherencia al tratamiento a nivel mundial y del $21 \%$ en 65 estudios que examinaron el tratamiento para el cáncer específicamen$t^{(23)}$. En un estudio realizado en Nnewi, Nigeria, se encontró que de 53 pacientes con diagnóstico de lesiones neoplásicas locales avanzadas a quienes se les realizó quimioterapia neoadyuvante (anterior a la cirugía), solo el $28.3 \%$ ( $n=15)$ completaron el tratamiento. De los 44 pacientes a los que se les ofreció quimioterapia adyuvante, solo el $38.6 \%(n=17)$ cumplieron con el tratamiento recomendado y solo 23 de estos pacientes completaron el tratamiento con radioterapia ${ }^{(24)}$.

Otros dos factores se han relacionado con la deserción del tratamiento oncológico: (1) la edad; $26 \%$ a $59 \%$ de adherencia en ancianos ${ }^{(25)} ; 40 \%$ a $60 \%$ en niños y adolescentes ${ }^{(17)} ;$ y (2) el momento del curso de la enfermedad en el que se administra el tratamiento ${ }^{(26)}$.

Para concluir, se destacan dos datos relevantes en general. El primero es que la tasa de no adherencia parece ser más baja con los medicamentos administrados por el personal de salud que con aquellos que son auto-administrados ${ }^{(17)}$. El segundo, que el porcentaje de adherencia en estudios de pacientes oncológicos está entre el $20 \%$ y el $100 \%{ }^{(27-28,66)}$.

Múltiples factores pueden modular la adherencia al tratamiento oncológico. En congruencia con el modelo biopsicosocial y cultural en el que se enmarca esta revisión, a continuación se presentan los factores biológicos, psicológicos, sociales y culturales que pueden aumentar la probabilidad de adherencia al tratamiento.

\section{FACTORES BIOLÓGICOS QUE AUMENTAN LA PROBABILIDAD DE ADHERENCIA}

Entre los factores biológicos que se han asociado con la adherencia al tratamiento se encuentran: (1) los aspectos específicos del mismo (tipo, complejidad, efectos secundarios y duración); (2) las características de la enfermedad (síntomas, duración, discapacidad y gravedad definida médicamente) ${ }^{(27)}$ y (3) el apoyo adecuado en el manejo de los efectos secundarios producidos por los tratamientos ${ }^{(4)}$.

En lo que se refiere a los aspectos específicos del tratamiento, se ha encontrado que la adherencia es mejor entre los pacientes que toman: (a) menor cantidad de medicamentos, (b) menor dosis del medicamento en cada administración, (c) me- 
dicamentos en forma de pastillas y no en forma líquida ${ }^{(17)}$.

Muchos pacientes prefieren la administración de los medicamentos antineoplásicos por vía oral, que por vía intravenosa por diversas razones: (a) la facilidad de administración, pues se evitan la inserción de un catéter venoso central, (b) la comodidad para el paciente y (c) la menor necesidad de hospitalización. En este caso los que determinan la adherencia son ellos mismos, no las enfermeras o los médicos como lo es cuando la administración es vía intravenosa ${ }^{(29)}$.

\section{FACTORES PSICOLÓGICOS QUE AUMENTAN LA PROBABILIDAD DE ADHERENCIA}

Entre los factores cognoscitivos que aumentan la probabilidad de adherencia se encuentran: (a) las actitudes generales que tienen los pacientes sobre la salud y la enfermedad ${ }^{(30)}$; (b) la percepción de riesgo, (c) los conocimientos ${ }^{(23,67)}$ específicamente los que se relacionan a las recomendaciones $^{(30)}$, (d) las creencias acerca de la salud y de los medicamentos ${ }^{(31-33,67)}$ y actitudes sobre el cuidado $^{(32,17)}$, (e) la experiencia previa que tienen los pacientes con los efectos secundarios de los tratamientos ${ }^{(34)}$ y (f) el optimismo ${ }^{(35-37)}$. En relación con este último factor, se ha descrito que el optimismo aumenta la percepción de calidad de vida y de bienestar subjetivo, disminuye el distrés, aumenta las esperanzas de sobrevivir y de poder controlar las recaídas ${ }^{(38)}$. Así mismo, la percepción de beneficio que tengan los pacientes en el tratamiento y el conocimiento acerca de los riesgos del tratamiento a seguir hacen que estos se adhieran a los mismos ${ }^{(39)}$.

En cuanto a los factores socioemocionales que pueden modular las conductas de adherencia, están: (a) las relaciones sociales, (b) la disposición emocional y (c) los estilos de regulación emocional, específicamente las creencias positivas acerca de la eficacia del tratamiento ${ }^{(23)}$. También se ha estudiado el efecto de la satisfacción en la adherencia encontrándose que es mejor en los pacientes que están satisfechos con el cuidado médico que están recibiendo. Algunos trabajos demuestran que pacientes que sienten que tienen el control sobre su salud (locus de control interno) pueden adherirse mejor, que los que sienten que su salud está más allá de su control (locus de control externo) ${ }^{(17)}$.

Las expectativas de un individuo pueden influir sustancialmente en los comportamientos de adherencia. Así lo confirman Partridge et al. ${ }^{(27)}$ cuando indican que una alta autoeficacia (expectativa de realizar correctamente una determinada conducta) se relaciona directamente con la creencia que tiene un paciente de que es capaz de adherirse y por tanto, exhibir conductas tales como tomar una pastilla, según la prescripción.

Antes del inicio del tratamiento, la conciencia y el sentimiento sobre la enfermedad y los posibles resultados, así como otras condiciones psicológicas o barreras (por ejemplo, las enfermedades mentales) pueden influir en la adherencia terapéutica y por lo tanto, deben ser reconocidos. No obstante, durante el tratamiento, las expectativas de resultado y los motivos individuales pueden cambiar debido a la percepción de la efectividad o de la toxicidad, lo que puede influir en la adherencia. Ello sugiere la necesidad de realizar un seguimiento cuidadoso ${ }^{(29)}$.

\section{FACTORES SOCIALES Y CULTURALES QUE AUMENTAN LA PROBABILIDAD DE ADHERENCIA}

Dentro de los factores sociales que aumentan la probabilidad de adherirse al tratamiento se encuentran: (a) el apoyo social y familiar ${ }^{(17,32)}$, (b) la cohesión familiar, 


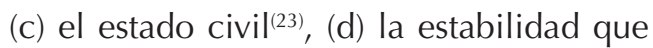
el grupo primario le dé al paciente ${ }^{(30)}$, (e) los grupos de apoyo ${ }^{(40,30)}$, (f) el grupo étni$\mathrm{CO}^{(41)}$; (g) la información del médico sobre el tratamiento a realizar, sobre los efectos secundarios y sobre el propósito del mismo ${ }^{(4)}$; (h) el tipo de trabajo (servicio o independiente); (i) nivel educativo superior y (j) estado civil casado ${ }^{(26)}$.

Se ha encontrado que dentro de los pacientes de avanzada edad, los de raza blanca presentan más adherencia a los medicamentos ${ }^{(25)}$. Sin embargo, este dato no está específicamente confirmado en pacientes oncológicos. No obstante, es evidente que los pacientes y los médicos que pertenecen al mismo grupo étnico son más propensos a compartir creencias culturales, valores y experiencias, que los hacen sentir mucho más cómodos entre sí y por ende incrementa la participación de los pacientes en su salud ${ }^{(41)}$.

Asimismo es importante que los pacientes se involucren en las discusiones acerca de su plan de tratamiento a través del diálogo abierto con los médicos, lo que a su vez favorece el empoderamiento en los pacientes $^{(67)}$. Y por esto uno de los factores relevantes en estos casos es la confianza en la relación médico-paciente ${ }^{(14)}$. La actitud y el interés del personal de salud en el paciente y las habilidades de comunicación juegan un rol importante en la adherencia. Es relevante que el personal de salud entienda las preocupaciones de los pacientes, incluyendo su diagnóstico y las preguntas acerca de la enfermedad y los medicamentos ${ }^{(17)}$. Se ha demostrado que una adecuada relación médico-paciente se asocia con mejoras en la participación del paciente en su cuidado, con disminución de cancelaciones a las visitas médicas e incremento en el compromiso del paciente con su tratamiento ${ }^{(30,41)}$.

\section{FACTORES BIOLÓGICOS QUE DISMINUYEN LA PROBABILIDAD DE ADHERENCIA}

Entre los factores biológicos que disminuyen la probabilidad de adherencia se encuentran: (1) los efectos secundarios de algunos tratamientos ${ }^{(4,5,17,18,23,30,43,66)} ;(2)$ deterioro en el estado funcional; (3) empeoramiento de la enfermedad ${ }^{(9)}$; (4) características del tratamiento, como por ejemplo, toxicidad especialmente si el paciente está tomando dosis muy seguidas o en un mal momento del día ${ }^{(21)}$; (5) el número de tabletas para ingerir cada día ${ }^{(67)}$ (6) la complejidad del régimen ${ }^{(68)}$ (7) edad; (8) ubicación del cáncer; (9) condición mental y física de los pacientes ${ }^{(38)}$ y (10) comorbilidad con otras enfermedades ${ }^{(67)}$.

Entre los efectos secundarios de algunos tratamientos se pueden mencionar por ejemplo: diarrea, pérdida de peso, alope$\mathrm{Cia}^{(23)}$ anorexia, insomnio, fatiga, vómito, nausea. Al respecto, Grunfeld et al. ${ }^{(33)}$ indican que la principal razón que tienen los pacientes con cáncer de mama para no tomar el Tamoxifeno son justamente los efectos secundarios que genera (síntomas urinarios, cansancio, sofocos, sudores nocturnos, entre otros). Así mismo Visram, Kanji y Dent ${ }^{(59)}$, en un estudio realizado con hombres diagnosticados con cáncer de mama, encontraron que de los 38 pacientes que recibieron Tamoxifeno, nueve de estos suspendieron su tratamiento debido a su toxicidad y se encontró que la causa más común fueron los sofocos producidos por este, además de disminución de la libido y aumento de peso. Lo mismo ocurrió con aquellos pacientes tratados con Letrozol pues debido a la toxicidad ninguno finalizó su tratamiento.

La edad es otro de los factores que disminuye la probabilidad de adherencia, aunque la relación no está totalmente clara. Según Patridge et al. ${ }^{(27,28)}$ la no adherencia se asocia con los extremos de la edad; 
pacientes con menos de 45 años y más de 75 u 85 son los menos adherentes ${ }^{(66,68)}$. La adherencia de los adultos mayores se ve influenciada significativamente por la toma de múltiples fármacos, así como por cuestiones psicosociales, incluido el apoyo social y la incidencia cada vez mayor de problemas de memoria. También Patridge et al. ${ }^{(28)}$, indican que las mujeres de menos de 45 años con cáncer de mama en estadios tempranos, se perciben menos invulnerables y por tanto, tienen mayor riesgo que las mujeres adultas mayores con este mismo diagnóstico de abandonar la quimioterapia oral.

En el estudio realizado por JanssenHeijnen et al. ${ }^{(60)}$ con pacientes con cáncer de pulmón de células pequeñas, se encontró que dentro de los motivos más frecuentes para no adherirse a los tratamientos oncológicos se encuentra tanto la negativa por parte de ellos y de sus familias, como la esperanza de vida corta o una combinación de edad avanzada, comorbilidad y pobre funcionalidad.

En lo que se refiere a la localización del cáncer, en un estudio realizado por Dinshaw et al. ${ }^{(26)}$ se encontró que contrario a lo esperado, las mujeres con cáncer de mama se adhieren más a la quimioterapia que aquellas con cáncer de cérvix, lo que se debe probablemente a la duración de la radioterapia en estas últimas.

\section{FACTORES PSICOLÓGICOS QUE DISMINUYEN LA PROBABILIDAD DE ADHERENCIA}

Algunos de los factores psicológicos que pueden favorecer la disminución de la adherencia al tratamiento son: (a) los estados emocionales negativos o la falta de motivación ${ }^{(21,42)}$; (b) los sentimientos negativos sobre la enfermedad ${ }^{(21)}$; (c) la baja autoeficacia, (d) las expectativas sobre los resultados $^{(21)}$, (e) las alteraciones psiquiátricas $(9,23,40,44,, 66)$, (f) la discapacidad, (g) la deficiencia de conocimientos sobre la enfermedad, sobre el tratamiento ${ }^{(23,9,44)}$ y sobre los resultados del mismo ${ }^{(23,9,44)}$, (h) discapacidad física causada por la enfermedad $^{(40)}$; (i) duración del tratamiento; (j) dosis del medicamento, $(\mathrm{k})$ dificultades para seguir el régimen terapéutico ${ }^{(29)}$ y (l) deterioro cognoscitivo ${ }^{(65)}$.

Se ha encontrado que el afecto o humor puede predecir la adherencia a la quimioterapia intravenosa ${ }^{(45)}$. En específico, algunos investigadores han indicado que la depresión y la ansiedad podrían afectar la voluntad del paciente y su capacidad para seguir adelante con el tratamiento $23,13,30,4)$. La depresión a menudo implica un grado apreciable de desesperanza(44) y el cumplimiento podría ser difícil o imposible para un paciente que tiene poco optimismo sobre el mérito de realizar cualquier acción ${ }^{(23,21,13)}$. De hecho, la depresión puede estar asociada con reducciones en el funcionamiento cognoscitivo ${ }^{(44)}$, esencial para recordar y seguir adelante con las recomendaciones del tratamiento (por ejemplo, tomar los medicamentos $)^{(13,68)}$. Además, la depresión clínica se asocia con el aislamiento social por el que suelen pasar los pacientes con enfermedades crónicas como el cáncer(44), mucho más cuando se presentan alteraciones con la imagen corporal, con las relaciones familiares $y$ con las relaciones sexuales ${ }^{(4)}$. En general, los costos emocionales del tratamiento oncológico pueden incluir la percepción de haber perdido el control de la situación ${ }^{(46)}$.

En un estudio realizado por Zhou et al. ${ }^{(47)}$ se encontró que la frecuencia y la extensión de las reacciones adversas a los medicamentos no están relacionadas con la depresión, pero sí lo está la experiencia subjetiva que el paciente tiene ante estas reacciones (anorexia, nausea y fatiga).

En cuanto a la ansiedad, es un factor precipitante para la exacerbación de efectos secundarios de los tratamientos, lo que disminuye la adherencia ${ }^{(8)}$. Específicamen- 
te la ansiedad que se presenta antes de una visita clínica, suele explicar que los pacientes no se adhieran por la anticipación de que tal visita podría estar asociada con recibir una mala noticia ${ }^{(48)}$. Aún con el conocimiento de que la intensión del tratamiento es curativa, los pacientes ansiosos tienden a discontinuarlo tempranamente ${ }^{(49)}$. No obstante, la asociación entre ansiedad y pobre adherencia es muy variable ${ }^{(23,13)}$. En coherencia con la respuesta ansiosa, el miedo es otro de los factores que influye en la pobre adherencia al tratamiento. El temor puede estar determinado por la anticipación o experiencia de dolor en la mamografía, el miedo a la radiación, el miedo de la hospitalización o de la cirugía ${ }^{(23)}$ y el miedo de perder el control o morir mientras se está bajo anestesia ${ }^{(4)}$. También el miedo está asociado a creencias fatalistas acerca del dolor por el cáncer, creencias negativas acerca de la efectividad de los medicamentos ${ }^{(14),}$ creencias relacionadas con que los tratamientos del cáncer no funcionan y que el cáncer es una sentencia de muerte por ser en esencia incurable (23,21). $^{2}$

Igualmente las creencias sociales o mitos sobre la salud pueden moderar la adherencia al tratamiento disminuyéndola o aumentándola. De hecho, las percepciones y creencias del paciente sobre la enfermedad y el pronóstico, son factores que interfieren en la adherencia al tratamiento(46). También está el caso de los pacientes que toman los medicamentos sólo cuando no se sienten bien y los suspenden en cuanto se sienten mejor o los síntomas

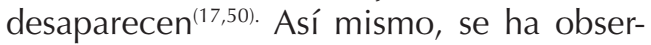
vado que los pacientes toman los medicamentos cuando consideran que si la dosis prescrita es buena, una dosis mayor va a ser mejor ${ }^{(50)}$, o los descontinúan porque los consideran ineficaces ${ }^{(17)}$. Respecto a este componente cognoscitivo de la adherencia, es oportuno mencionar que la interpretación que hacen los pacientes del significado de un régimen terapéutico y el desarrollo cognoscitivo están asociados. Por ejemplo, Thu Ma et al. ${ }^{(51)}$ encontraron que los pacientes de edad avanzada con cáncer de seno, tienen menor probabilidad de adherirse a la quimioterapia y a la radioterapia debido a que perciben disminuida su calidad de vida durante esos tratamientos. De hecho, las mujeres más jóvenes tienden a ser menos adherentes, en específico al uso del Tamoxifeno, posiblemente porque se sienten menos vulnerables y por el hecho de que no están acostumbradas a tomar medicamentos a diario, como sí lo están posiblemente mujeres de edad más avanzada.

Justamente, en referencia a los medicamentos orales como el Tamoxifeno, se ha reportado que los siguientes factores psicológicos están asociados con la no adherencia: solicitudes de cambios conductuales sustanciales, insatisfacción con el cuidado recibido e historia de enfermedad mental y de no adherencia ${ }^{(27)}$. Sin embargo, las tasas de no adherencia a la quimioterapia oral son ligeramente mayores que las de quimioterapia intravenosa. Es posible que se deba que los medicamentos orales tomados en casa o auto-administrados, no son directamente monitoreados por los médicos, lo cual hace que exista menos control en la toma de los mismos ${ }^{(18)}$.

Pero sin lugar a dudas, lo que Tebbi ${ }^{(17)}$ denomina errores humanos (el olvido, las dificultades para aprenderse y recordar las instrucciones, la preocupación y el inadecuado suministro de los medicamentos) constituyen la causa más importante para la no adherencia al tratamiento en niños y en adolescentes. Al respecto, Partridge ${ }^{(19)}$ indica que mujeres con cáncer de seno que reciben quimioterapia oral reportan no adherirse al tratamiento simplemente porque se les olvida o porque deciden no querer tomar las medicinas. Sin embargo, los regímenes de medicación de una sola dosis generan tasas significativamente más 
altas de adherencia que aquellos con múltiples dosis, sin embargo hay que tener en cuenta que no solo es el número de dosis sino también el número de fármacos a utilizar (29).

En lo que se refiere específicamente a la duración del tratamiento, se ha encontrado que cuando los regímenes terapéuticos implican cambios en los hábitos comportamentales (por ejemplo: tomar medicamentos, dejar de fumar, reducir el consumo del alcohol) se produce una baja adherencia en los pacientes y por ende, entre más complejo sea el régimen recomendado, mayor será el nivel de incumplimiento por parte del paciente $\mathrm{e}^{(29,30,33)}$.

\section{FACTORES SOCIALES Y CULTURALES QUE DISMINUYEN LA PROBABILIDAD DE ADHERENCIA}

Entre los factores sociales y culturales que influyen en la pobre adherencia al tratamiento se pueden destacar: (1) el conflicto familiar (falta de ayuda y de apoyo por parte de los miembros de la familia y de amigos) $^{(13,44)}$; (2) la comunicación deficiente con amigos y parientes ${ }^{(23)}$; (3) el aislamiento social ${ }^{(30)}$; (4) la mala o inadecuada comunicación médico-paciente ${ }^{(4,9,20,21)}$; (5) el incremento del consumo de recursos de la salud, como, por ejemplo, más visitas al médico, mayores tasas de hospitalización y estancias más largas ${ }^{(19,20,21,27)}$; (6) oportunidad de cita; esto es, problemas para obtener una cita médica, como por ejemplo tener que esperar mucho tiempo para que se la den ${ }^{(39)}$; (7) los altos costos de los medicamentos ${ }^{(27,68)}$.

Por ejemplo, Balkrishnan ${ }^{(25)}$ ha demostrado que hay mayores tasas de incumplimiento con el tratamiento en los ancianos cuando los costos de los medicamentos son elevados; (8) la situación socioeconómica $^{(13,18,21,43)}$; (9) factores sociodemográficos como edad, raza y etnia ${ }^{(9,20,21,41)} ;$ y (10) el nivel educativo ${ }^{(14,40)}$.
Concretamente se ha encontrado que los problemas en la comunicación tienen un impacto en el proceso de la toma de decisiones en los pacientes con cáncer de mama ${ }^{(52)}$. La falta de información acerca de las ventajas y desventajas del tratamiento es algo que influye notablemente en que los pacientes no se adhieran al tratamiento al igual que cuando los beneficios de éste no son aparentemente obvios para el paciente $^{(5)}$.

En lo que se refiere a la edad, se afirma que los adolescentes son menos adherentes que los pacientes pediátricos ${ }^{(17,21)}$. Se ha clasificado a los adolescentes como el grupo etario menos adherente, debido a que tienen una comprensión menos desarrollada de su enfermedad, menor vulnerabilidad percibida ${ }^{(69)}$, niveles más altos de negación ${ }^{(27)}$, comportamiento rebelde y desacuerdo con los padres y con las figuras de autoridad, a diferencia de los niños o de los pacientes más jóvenes que por lo general reciben los medicamentos bajo el control de los padres ${ }^{(34)}$. Por otra parte, hay menor apoyo social en pacientes de más edad que en pacientes jóvenes ${ }^{(15,27)}$, especialmente en aquellos con más de 85 años ${ }^{(28)}$ lo que influye directamente en la adherencia al tratamiento. La edad avanzada puede representar un obstáculo para la adecuada adherencia a la quimioterapia oral, pues probablemente se consumen múltiples medicamentos a la vez ${ }^{(29)}$.

En cuanto al grupo étnico, el personal de salud mental en general no ha sido sensible a las diferencias étnicas en cuanto a la forma en que los pacientes reconocen, definen y expresan sus síntomas y esto ha hecho que ellos tengan una actitud negativa hacia el personal de salud, lo que puede causar retraso en la búsqueda de atención o seguimiento y la no adherencia al tratamiento médico como tal ${ }^{(41)}$. Específicamente se ha encontrado que los pacientes de raza negra presentan menos probabilidad de adherencia que los pa- 
cientes de raza blanca en iniciar una terapia adyuvante en el tratamiento de cáncer de seno o de cáncer colorectal. Sin embargo otros investigadores han encontrado tales asociaciones entre la adherencia al tratamiento y la raza, son inconsistentes ${ }^{(9)}$.

Otro de los factores que se ha encontrado que influye en la no adherencia al tratamiento son las creencias supersticiosas en el uso de los enfoques tradicionales de curación y / o espirituales ${ }^{(43)}$.

La adherencia a una rehabilitación adecuada después de la cirugía oncológi$\mathrm{ca}$, que suele incluir sesiones de radioterapia y ciclos de quimioterapia, se puede ver afectada por factores psicosociales como; alcoholismo, situación familiar caótica o circunstancias que pueden limitar el acceso al tratamiento como problemas económicos o distancia geográfica ${ }^{(4)}$.

\section{MÉTODOS Y TÉCNICAS DE EVALUACIÓN E INTERVENCIÓN PARA LOS PROBLEMAS DE ADHERENCIA AL TRATAMIENTO ONCOLÓGICO}

La literatura describe diversas formas de evaluar la adherencia al tratamiento en general y es menos específica con respecto a la quimioterapia y/o radioterapia aunque para ello se ha sugerido el uso de estrategias genéricas como: (a) entrevistas longitudinales de los pacientes, (b) cuestionarios estandarizados y (c) entrevistas a médicos ${ }^{(18)}$. En general, los métodos de evaluación de la adherencia se pueden clasificar en: (a) medidas directas de adherencia; por ejemplo, marcadores en la orina, niveles séricos de los medicamentos y de sus metabolitos ${ }^{(17,40,54) ;}$ (b) medidas indirectas como número de pastillas o monitores electrónicos de conteo de pastillas y (c) medidas subjetivas como reportes de los pacientes $\mathrm{u}$ otras personas ${ }^{(53)}$, tales como los auto-reportes ${ }^{(22,29,44,55)}$; y los diarios ${ }^{(55)}$. Además, se sugiere hacer uso de la historia clínica ${ }^{(44)}$ y el recuerdo del médico sobre la prescripción hecha ${ }^{(22)}$. Así mismo, para evaluar la adherencia se tienen en cuenta las medidas de no auto-reporte o medidas indirectas, tales como: (a) reclamos administrativos, (b) conteo de las pastillas o peso del frasco, (c) monitores electrónicos y (d) la opinión clínica ${ }^{(55)}$.

Dentro de los métodos y técnicas de intervención utilizados para la adherencia de los pacientes a los tratamientos es relevante mencionar tres tipos de intervenciones: (1) intervenciones pedagógicas o educativas $^{(21,48,54)}$, las cuales pueden impartirse de manera verbal o escrita, individual o grupalmente. A menudo se utilizan materiales audiovisuales o enviados por correo e instrucciones por teléfono. Hoy en día los celulares son un método popular que se está usando para ayudar a los pacientes a recordar la toma de sus medicamentos, al igual que los mensajes de texto; también algunos pacientes reportan el uso de relojes de alarma o temporizadores de cocina $^{(67) ;}$ sin embargo hay que tener en cuenta que estos métodos no son tan efectivos con personas de mayor edad ya que no dominan el uso de esta tecnología o no están dispuestos a utilizarla ${ }^{(70)}$. (2) Intervenciones centradas en el comportamiento, las cuales tienen como objetivo orientar, formar o reforzar patrones específicos del comportamiento. (3) Estrategias enfocadas afectivamente con el objetivo de influir en la adherencia a través de relaciones sociales y apoyo social, incluyendo el soporte familiar y las visitas domiciliarias. Se ha demostrado que cuando se combinan este tipo de intervenciones, el resultado es mucho más efectivo que utilizarlas de manera independiente ${ }^{(53)}$. Es importante tener en cuenta que en pacientes oncológicos un seguimiento a largo plazo es lo más importante debido a la cronicidad de la enfermedad ${ }^{(67)}$. Sin embargo, no se encontró referencia de estrategias específicamente diseñadas para la adherencia a la quimioterapia y a la radioterapia, aunque se 
propone utilizar la educación y estrategias motivacionales sustentadas en el Modelo de Creencias en Salud(67), que promuevan la adherencia a través del incremento en la motivación. Por su parte, Blondin et al. ${ }^{(70)}$ Encontraron en su investigación con pacientes con cáncer de cabeza y cuello que la adherencia fue más alta con el uso de diarios de papel que con sistemas de respuesta de voz interactivos.

Pero aunque existen diversidad de intervenciones dirigidas a incrementar la adherencia al tratamiento de enfermedades crónicas como la hipertensión, la diabetes, el asma, el Virus de la Inmunodeficiencias Humana (VIH), la artritis reumatoide y la epilepsia $^{(40)}$, no se hallaron protocolos específicos para aumentar la adherencia en pacientes oncológicos que reciben quimioterapia y/o radioterapia.

\section{DISCUSIÓN Y CONCLUSIONES}

Con base en la revisión previamente presentada, se puede concluir que la adherencia al tratamiento oncológico es el complejo de conductas que los pacientes con cáncer adquieren y mantienen a partir del momento en que deciden seguir las instrucciones y prescripciones terapéuticas, bien sea en relación con la quimioterapia y/o la radioterapia, y participar activamente junto con el oncólogo en la toma de decisiones sobre su tratamiento y el efecto que éste tendrá en su calidad de vida. Por tanto, el hecho de que los pacientes no se adhieran a los tratamientos oncológicos conlleva a que pongan en peligro su salud, incluso exponiendo su vida. La mayoría de las definiciones de adherencia $^{(21,40,56,57)}$, resaltan como elemento principal el cumplimiento (realizar el número preciso de sesiones, durante el tiempo, con la periodicidad y con la frecuencia indicados), ignorando la importancia del rol activo del paciente en el proceso terapéutico.
De acuerdo con lo anterior, se propone considerar a la adherencia como un comportamiento de alta complejidad que resulta entre otros de la motivación, la capacidad de tomar decisiones, la autonomía y la filosofía de vida de cada paciente e implica tanto el cumplimiento como el compromiso y la responsabilidad de asumir una opción terapéutica. De hecho, así lo confirma Perestelo-Pérez ${ }^{(58)}$ al indicar que el concepto de "toma de decisiones compartidas" (TDC) surge en contextos donde pacientes y profesionales sanitarios han de tomar decisiones sobre pruebas diagnósticas o tratamientos en los que no se dispone de una única o "mejor opción". Esto implica definir la adherencia en términos de lo que el paciente está convencido y seguro de hacer y no en función de lo que no hace ${ }^{(8,9)}$. Igualmente varios autores $^{(4,9,14,20,21,30,42)}$ coinciden en la importancia de una relación adecuada entre el médico y el paciente, mucho más si se considera que la adherencia es un término que incluye intervención tanto del paciente como del profesional de la salud para alcanzar a cabalidad el objetivo terapéutico propuesto.

En cuanto a las tasas de deserción al tratamiento oncológico, se encontró una franca deficiencia de datos a nivel nacional. Por lo tanto, parece pertinente realizar investigación que permita determinar las tasas de adherencia a este tratamiento y así mismo, las de deserción. Mucho más si se considera que un paciente que se adhiere, es un paciente con una mayor probabilidad de aumentar su tiempo de sobrevida. Además, la deserción del tratamiento implica altos costos biológicos, psicológicos y sociales posteriores para el paciente y por supuesto, algunos de carácter económico para el sistema de salud. Así mismo, los datos sobre tasas de deserción y adherencia a nivel mundial son poco concluyentes, aunque hay mayor evidencia empírica. Específicamente en tratamientos 
oncológicos (quimioterapia y radioterapia) prolongados, la adherencia es menor (cerca del $21 \%)^{(23)}$ y está asociada a una compleja multitud de factores. Entre estos, están: (a) los efectos secundarios característicos de la quimioterapia y la radioterapia $^{(4,5,17,18,23,30,33,43,59)}$; (b) la subjetividad de la experiencia con la que el paciente viva cada momento del curso de la enfermedad y del tratamiento oncológico; (c) la percepción de control; (d) la evaluación de la importancia de adherirse al tratamiento y de las consecuencias de no hacerlo; (e) la claridad en los conocimientos que tengan acerca de su enfermedad y su tratamiento; (f) la percepción autoeficacia.

Diferentes autores coinciden en la gran influencia que tienen algunas alteraciones psiquiátricas, como la depresión y la ansiedad, en la baja adherencia al tratamiento pues en ocasiones la desesperanza, el aislamiento social, los temores que presentan algunos pacientes impiden que adquieran una nueva conducta como es la de adherirse a la quimioterapia o a la radioterapia. Además es importante tener en cuenta que la ansiedad en muchas ocasiones exacerba una maximización de los efectos secundarios de los tratamientos ${ }^{8}$, por lo cual sería necesario controlar esta variable para que así los pacientes no interrumpan su tratamiento. Esto se reafirma en la importancia del apoyo social y familiar para los pacientes oncológicos a lo largo de su proceso de enfermedad y por ende al momento de adherirse al tratamiento indicado por el médico. Específicamente se ha encontrado que la falta de relaciones sociales y la pobre comunicación tanto familiar como con el profesional de la salud son factores que influyen notablemente en la no adherencia al tratamiento por parte de los pacientes oncológicos.

Por otra parte es importante resaltar que los datos reportados en la literatura revisada no son concluyentes con respecto a cuál grupo etario es más o menos adhe- rente. Lo cierto es que ante el asunto de la edad y la adherencia, debe considerarse que la decisión final de aceptar un tratamiento y promover el cumplimiento al mismo, está en manos de los padres de pacientes menores de edad. La pregunta que subyace a ello es: ¿En realidad están adheridos a su tratamiento oncológico estos niños y adolescentes a quienes sus padres o representantes legales les exigen cumplir con las prescripciones del médico tratante?

En lo que se refiere a la evaluación y la intervención psicológicas de la adherencia, se encuentra un mayor déficit en la exactitud de la primera y una mayor producción e interés en la segunda. Aunque existe un amplio repertorio de auto-reportes bastante utilizados según lo indica la literatura; la deficiencia de éstos para medir la adherencia al tratamiento oncológico, es evidente. Además, los instrumentos de carácter cuantitativo disponibles son superados por la subjetividad y por ende, los datos que arrojan no son altamente confiables. En consecuencia, parece relevante realizar futuras investigaciones en este campo. En cuanto a las intervenciones psicológicas para aumentar la adherencia de los pacientes a los tratamientos oncológicos, se puede concluir que es pertinente que incluyan tanto factores pedagógicos, como modificaciones en el comportamiento $y$ estrategias enfocadas en el afecto ya que se ha comprobado que haciéndolo de esta manera el proceso terapéutico es mucho más efectivo. No obstante, al igual que en el aspecto evaluativo, no hay referencia en la literatura revisada a intervenciones protocolizadas desde el modelo cognitivo comportamental para aumentar la adherencia al tratamiento oncológico. Y por el contrario vale la pena resaltar la cantidad de programas desarrollados, dirigidos en su mayoría por personal de enfermería.

Con base en lo anterior es importante hacer énfasis en la falta de investigación psicológica en lo que respecta a la adhe- 
rencia a la quimioterapia y/o radioterapia en pacientes con cáncer específicamente, pues como se resaltó en este artículo estos tipos de tratamientos tienen un gran componente psicosocial y biológico, así como determinados factores que influyen tanto a favor como en contra de la misma. Desde la psicología se podría abordar este tema y favorecer el cumplimiento de la adherencia debido al porcentaje significativo de pacientes que no lo hacen. Por lo cual se recomienda realizar intervenciones psicológicas que incrementen la adherencia de estos pacientes a los tratamientos propuestos y que hagan parte del manejo interdisciplinario e integral que deben recibir. Es posible que estas intervenciones psicológicas incluyan entre otros, algunos elementos esenciales como la educación, el entrenamiento en toma de decisiones y solución de problemas, la reestructuración de ideas irracionales y manejo de creencias, actitudes y atribuciones que interfieran con la adherencia, el manejo del estrés y de la frustración, entrenamiento en autocontrol, manejo de emociones perturbadoras y psicopatología, así como entrenamiento en asertividad y comunicación para la promoción del establecimiento de una relación médico-paciente efectiva. En conclusión, se propone que la Psicología tenga una participación más activa en el manejo de problemas relacionados con la adherencia terapéutica y que ello no esté delegado en el personal médico y de enfermería. En congruencia con la propuesta de Valentín et al.(61), esto haría parte de la atención integral de la persona enferma en su realidad total (cuidados continuos) pues si bien el tratamiento sintomático suele ser el eje central de la intervención, el tratamiento de las necesidades psicológicas y sociales también ocupan un lugar fundamental en el proceso de enfermedad.

\section{REFERENCIAS BIBLIOGRÁFICAS}

1. Fleming ID, Braddy LW, Mielszkalski GB, Cooper MR, Cooper MR. Bases de los principales tratamientos actuales del cáncer. En: Murphy GP, Lawrence Wjr, Lenhard RE. Oncología clínica. Washington DC: Organización Panamericana de la Salud (OPS) - American Cancer Society. 1996; p. 110-53.

2. Díaz-Rubio E. Aspectos generales de terapéutica oncológica. En Die Goyanes $A$, Llombart Bosh M, Matilla Vicente A, editores. Manual de oncología básica. Madrid: Asociación española contra el cáncer, 2000; p. 69-77.

3. Buela-Casal G, Moreno S. Intervención psicológica en cáncer. En: Simón MA, editor. Manual de Psicología de la Salud. Fundamentos, metodología y aplicaciones. Madrid: Biblioteca Nueva, 1999; p. 505-35.

4. Holland J. Psycho-Oncology. New York: Oxford University Press; 1998.

5. Atkins L, Fallowfield L. Intentional and nonintentional non-adherence to medication amongst breast cancer patients. Eur J Cancer 2006; 42 (14): 2271-76. Doi:10.1016/j. ejca.2006.03.004

6. Robb KA, Williams JE, Duvivier V, Newhan DJ. A pain management program for chronic cancer treatment related pain: a preliminary study. J Pain 2006; 7 (2): 82-90. Doi:10.1016/j.jpain.2005.08.007

7. Haynes RB. Introduction. En: Haynes RB, Taylor DW, Sackett DL, eds. Compliance in health care Baltimore: John Hopkins University Press; 1979. p. 1-7.

8. Greer J, Pirl W, Park E, Lynch T, Temel J. Behavioral and psychological predictors of chemotherapy adherence in patients with advanced non-small cell lung cancer. J Psychosom Res 2008; 65 (6): 549-52. Doi:10.1016/j.jpsychores.2008.03.005

9. Neugut A, Matasar M, Wang X, McBride R, Jacobson J, Tsai W, Grann V, Hershman D. Duration of Adjuvant Chemotherapy for Colon Cancer and Survival Among the El- 
derly. J Clin Oncol 2006; 24 (15): 2368-75. Doi: $10.1200 / \mathrm{JCO} .2005 .04 .5005$

10. Burrell A, Wong P, Ollendorf D, et al. Defining compliance/adherence and persistence: ISPOR Special Interest Working Group. Value Health 2005; 8:A194-5.

11. Alfonso L. Acerca del concepto de adherencia terapéutica. Rev Cubana de Salud Pública 2004; 30 (4): 350-52.

12. Brannon L, Feist J, editores: Psicología de la salud. Madrid: Thomson; 2001.

13. DiMatteo MR, Lepper H, Croghan T. Depression is a risk factor for noncompliance with medical treatment. Arch Intern Med 2000; 160 (14): 2101-7.

14. Jacobsen R, Møldrup C, Christrup L, Sjøgren $\mathrm{P}$, Hansen $\mathrm{O}$. The Danish version of the Medication Adherence Report Scale: Preliminary Validation in Cancer Pain Patients. Pain Pract 2009; 9 (1): 1-7. Doi: 10.1111/j.1533-2500.2008.00245.x

15. Maio E, Gravina A, Pacilio C, Amabile G, Labonia V, Landi G, et al. Compliance and toxicity of adjuvant CMF in elderly breast cancer patients: A single-center experience. BMC Cancer 2005; 5 (30): 1-10. Doi:10.1186/1471-2407-5-30

16. Szajnberg N, Altman A, Quinn J, Weisman $\mathrm{S}$. Physician/Nurse attitudes and treatment adherence in children with cancer: A pilot study and new instrument. Psychooncology 1995; 4 (1): 47-53. Doi: 10.1002/ pon. 2960040106

17. Tebbi C. Treatment compliance in childhood and adolescence. Cancer 1993; 71 (Suppl 10): 3441-9. Doi: 10.1002/10970142

18. Lebovits A, Strain J, Schleifer S, Tanaka J, Bhardwaj S, Messe M. Patient noncompliance with self-administered chemotherapy. Cancer 1990; 65 (1): 17-22. Doi: 10.1002/1097

19. Partridge $\mathrm{AH}$. Non-adherence to endocrine therapy for breast cancer. Ann Oncol 2006; 17 (2): 183-84.

20. Partridge $\mathrm{AH}$, LaFountain A, Mayer E, Taylor B, Winer E, Asnis-Alibozek, A. Adhe- rence to initial adjuvant anastrozole therapy among women with early-stage breast cancer. J Clin Oncol 2008; 26 (4): 556-62. Doi: 10.1200/JCO.2007.11.5451

21. Ruddy K, Mayer E, Partridge A. Patient adherence and persistence with oral anticancer treatment. CA Cancer J Clin 2009; 59 (1): 56-66. Doi:10.3322/caac.20004.

22. Ruddy K, Partridge AH. Adherence with adjuvant hormonal therapy for breast cancer. Ann Oncol 2009; 20 (3): 401-2. Doi: 10.1093/annonc/mdp039

23. Magai C, Consedine N, Neugut A, Hershman D. Common psychosocial factors underlying breast cancer screening and breast cancer treatment adherence: A conceptual review and synthesis. J Womens Health (Larchmt) 2007; 16 (1): 11-23. Doi: 10.1089/jwh.2006.0024.

24. Anyanwu SNC, Egwuonwu OA, Ihekwoaba EC. Acceptance and adherence to treatment among breast cancer patients in Eastern Nigeria. Breast 2011; 20 (2): S51S53. Doi: 10.1016/j.breast.2011.01.009

25. Balkrishnan R. Predictors of Medication Adherence in the Elderly. Clin Ther 1998; 20 (4): 764-71. Doi:10.1016/S01492918(98)80139-2

26. Dinshaw K, Mishra G, Shastri S, Badwe R, Kerkar R, Ramani R, et al. Determinants of compliance in a cluster randomised controlled trial on screening of breast and cervix cancer in Mumbai, India. Oncology 2007; 73 (3-4): 154-61. Doi: 10.1159/000126498

27. Partridge AH, Avorn J, Wang P, Winer E. Adherence to therapy with oral antineoplastic agents. J Natl Cancer Inst 2002; 94 (9): 652-61. Doi: 10.1093/jnci/94.9.652

28. Partridge $\mathrm{AH}$, Wang $\mathrm{P}$, Winer $\mathrm{E}$, Avorn J. Nonadherence to adjuvant tamoxifen therapy in women with primary breast cancer. J Clin Oncol 2003; 21 (4): 602-6. Doi: 10.1200/jco.2003.07.071

29. Banna GL, Collovà E, Gebbia V, Lipari H, Giuffrida P, Cavallaro S, et al. Anticancer oral therapy: Emerging related issues. Can- 
cer Treat Rev 2010; 36(8): 595-605. Doi: 10.1016/j.ctrv.2010.04.005

30. Rodríguez-Marín J. Psicología social de la salud. Madrid: Editorial Sintesis; 1995.

31. Horne R, Weinman J. Patient's beliefs about prescribed medicines and their role in adherence to treatment in chronic physical illness. J Psychosom Res 1999; 47 (6): 55567.

32. Kissane D, Grabsch B, Clarke D, Christie G, Clifton D, Gold S, et al. Supportive expressive group therapy: The transformation of existential ambivalence into creative living while enhancing adherence to anti-cancer therapies. Psychooncology 2004; 13 (11): 755-68. Doi: 10.1002/pon.798

33. Grunfeld E, Hunter M, Sikka P, Mittal S. Adherence beliefs among breast cancer patients taking tamoxifen. Patient Educ Couns 2005; 59 (1): 97-102. Doi: 10.1016/j. pec.2004.10.005

34. Lehrnbecher $T$, Laws $H$, Boehm A, Dworzak M, Janssen G, Simon A y Groll A. Compliance with anti-infective preventive measures: A multicentre survey among pediatric oncology patients. Eur J Cancer 2008; 44 (13): 1861-65. Doi:10.1016/j. ejca.2008.06.022

35. Epping-Jordan JE, Compas BE, Osowiecki DM, Oppedisano G, Gerhardt C, Primo K, Krag DN. Psychological adjustment in breast cancer: Processes of emotional distress. Health Psychol 1999; 18(4): 315-26. Doi: 10.1037/0278-6133.18.4.315

36. Carver CS, Lehman JM y Antoni MH. Dispositional pessimism predicts illness-related disruption of social and recreational activities among breast cancer patients. J Pers Soc Psychol 2003; 84(4): 813-21. Doi: 10. 1037/0022-3514.84.4.813

37. Godin G, Cote J, Naccache H, Lambert LD, Trottier S. Prediction of adherence to antiretroviral therapy: A one-year longitudinal study. AIDS Care 2005; 17(4): 493-504. Doi: 10.1080/09540120412331291715

38. Ramfelt E, Lützén K. Patients with cancer: Their approaches to participation in treatment plan decisions. Nurs Ethics 2005; 12 (2): 143-55. Doi: 10.1191/0969733005ne771 oa

39. Zheng Y, Saito T, Takahashi M, Ishibashi T, Kai I. Factors associated with intentions to adhere to colorectal cancer screening follow-up exams. BMC Public Health 2006; 6 (272): 1-12. Doi: 10.1186/1471-2458-6272

40. McDonald H, Garg A, Haynes B. Interventions to enhance patient adherence to medication prescriptions. JAMA 2002; 288 (22): 2868-79. Doi:10.1001/jama.288.22.2868.

41. Kennedy BR, Mathis CC, Woods AK. African americans and their distrust of the health care system: Healthcare for diverse populations. J Cult Divers. 2007; 14(2):5660.

42. Ortiz M, Ortiz E. Psicología de la salud: Una clave para comprender el fenómeno de la adherencia terapéutica. Rev Méd Chile 2007; 135 (5): 647-52.

43. Adisa A, Lawal O, Adesunkanmi A. Evaluation of patients' adherence to chemotherapy for breast cancer. Afr J Health Sci 2008; 15 (1): 22-27. Doi:10.4103/09731482.42640

44. DiMatteo MR, Giordani PJ, Lepper HS, Croghan TW. Patient adherence and medical treatment outcomes. Med Care 2002; 40 (9): 794-811. Doi: 10.1097/01. MLR.0000024612.61915.2D.

45. Ayres A, Hoon PW, Franzoni JB, Matheny KB, Cotanch PH, Takayanagi S. Influence of mood and adjustment to cancer on compliance with chemotherapy among breast cancer patients. J Psychosom Res 1994; 38 (5): 393-402.

46. Richardson JL, Sanchez K. Compliance with cancer treatment. En: Holland JC. Psycho-Oncology. New York: Oxford University Press; 1998. p. 67-77.

47. Zhou T, Duan JJ, Zhou GP, Cai JY, Huang, $\mathrm{ZH}$, Zeng, YT, Xu F. Impact of depression mood disorder on the adverse drug reaction incidence rate of anticancer drugs in cancer patients. J Int Med Res 2010; 38 (6): 2153-59. 
48. Cardella J, Coburn N, Gagliardi A, Maier BA, Greco E, Last L, et al. Compliance, attitudes and barriers to post-operative colorectal cancer follow-up. J Eval Clin Pract 2008; 14 (3): 407-15. Doi:10.1111/j.13652753.2007.00880.x

49. Thomas B, Thomas I, Nandamohan V, Nair M, Pandey M. Screening for distress can predict loss of follow-up and treatment in cancer patients: Results of development and validation of the Distress Inventory for Cancer Version 2. Psychooncology 2009; 18 (5), 524-33. Doi: 10.1002/pon.1422

50. Peralta M. Adherencia a tratamiento. Rev Cent Dermatol Pascua 2008; 17(3): 84-8.

51. Thu Ma A, Barone J, Wallis A, Jade Wu N, Garcia L, Estabrook A, Rosenbaum-Smith S, Tartter P. Noncompliance with adjuvant radiation, chemotherapy, or hormonal therapy in breast cancer patients. Am J Surg 2008; 196 (4): 500-04. Doi:10.1016/j.amjsurg.2008.06.027

52. Beisecker A, Cook M, Ashworth J, Hayes J, Brecheisen $M$, Helmig $L$, et al. Side effects of adjuvant chemotherapy: Perceptions of node--negative breast cancer patients. Psychooncology 1997; 6 (2): 85-93. Doi:10.1002/ (SICI)1099-1611(199706)6:2<85::AIDPON247>3.0.CO;2-T

53. Roter DL, Hall JA, Merisca R, Ordstrom $B$, Cretin D, Varsta B. Effectiveness of interventions to improve patient compliance. Med Care 1998; 36 (8): 1138-61.

54. Richardson JL, et al. The Effect of compliance with treatment on survival among patients with hematologic malignancies. J Clin Oncol 1990; 8 (2): 356-64.

55. Garber M, Nau D, Erickson S, Aikens J y Lawrence J. The concordance of self-report with other measures of medication adherence: A summary of the literature. Med Care 2004; 42 (7): 649-52. Doi: 10.1097/01. mlr.0000129496.05898.02

56. Dilla T, Valladares A, Lizan L, Sacristan J. Adherencia y persistencia terapéutica: causas, consecuencias y estrategias de mejora.
Atención primaria 2009; 41 (6): 342-48. Doi:10.1016/j.aprim.2008.09.031

57. Kondryn HJ, Edmondson CL, Hill JW, Eden $\mathrm{T}$. Treatment non-adherence in teenage and young adult cancer patients: A preliminary study of patient perceptions. Psychooncology 2009; 18 (12), 1327-32. Doi: 10.1002/ pon.1541

58. Perestelo-Pérez L, Pérez-Ramos J, RiveroSantana A, González-Lorenzo M. Toma de decisiones compartidas en Oncología. Psicooncología 2010; 7(2-3): 233-42.

59. Visram H, Kanji F, Dent SF. Endocrine therapy for male breast cancer: rates of toxicity and adherence. Curr Oncol 2010; 17 (5): 17-21. Doi:10.3747/co.v17i5.631

60. Janssen-Heijnen ML, Maas HA, Van de Schans SA, Coeberg JW, Groen HJ. Chemotherapy in elderly small-cell lung cancer patients: yes we can, but should we do it? Ann Oncol 2011; 22 (4): 821-6. Doi:10.1093/annonc/mdq448

61. Valentín V, Camps C, Carulla J, Casas A, González-Barón, M. La organización de los cuidados continuos. Psicooncología 2004; 1 (2-3): 7-21.

62. Martín M, López S. Tratamiento de la emesis inducida por citotóxicos. Psicooncología 2004; 1 (2-3): 117-34.

63. Vega ME, De Juan A, García A, López JM, López C, López A, Rivera F, Ramos J. Aspectos psicológicos de la toxicidad de la quimioterapia. Psicooncología 2004; 1 (23): 137-50.

64. Murillo MT, Valentín V, Valentín M. Síntomas asociados al tratamiento con radioterapia. Psicooncología 2004; 1 (2-3): 151168.

65. Rubio B, Sirgo A, Forcadell E, Mele M, Guma J. Deterioro cognitivo inducido por los tratamientos oncológicos sistémicos en cáncer de mama no metástico: revisión de estudios. Psicooncología 2009; 6 (1): 83121.

66. Verma S, Madamas Y, Sehdev S, Martin G, Bajcar J. Patient adherence to aromatase inhibitor treatment in the adjuvant setting. 
Curr Oncol 2011; 8 (1): 3-9. Doi:10.3747/ co.v17i5.631

67. Schneider SM, Hess K, Gosselin T. Interventions to promote adherence with oral agents. Sem Oncol Nurs 2011; 27 (2): 133141. Doi:10.1016/j.soncn.2011.02.005

68. Hadji P. Improving compliance and persistence to adjuvant tamoxifen and aromatase inhibitor therapy. Crit Rev Oncol Hematol 2010; 73: 156-66. Coi:10.1016/j.critrevonc.2009.02.001

69. Kondryn HJ, Edmondson CL, Hill J, Eden T. Treatment non-adherence in teenage and young adult patients with cancer. Lancet
Oncol 2011; 12: 100-08. Doi:10.1016/ S1470- 2045(10)70069-3.

70. Blondin JM, Abu-Hasaballah KS, Tennen H, Lalla RV. Electronic versus paper diaries: A pilot study of concordance and adherence in head and neck cancer patients receiving radiation therapy. Head Neck Oncol 2010; 2: 29. Doi:10.1186/1758-3284-2-29

71. Blesser S, Schwartzberg L, Husain N, Johnsrud M. Patient and plan characteristics affecting abandonment of oral oncolytic prescriptions. Am J Manag Care 2011; 17 (5 Spec No.):SP38-SP44. Doi: 10.1200/ JOP.2011.000316 